\title{
Duo property for rings by the quasinilpotent perspective
}

\author{
Harmanci A. ${ }^{1}$, Kurtulmaz Y. $^{2}$, Ungor B. ${ }^{3}$
}

\begin{abstract}
In this paper, we focus on the duo ring property via quasinilpotent elements, which gives a new kind of generalizations of commutativity. We call this kind of rings qnil-duo. Firstly, some properties of quasinilpotents in a ring are provided. Then the set of quasinilpotents is applied to the duo property of rings, in this perspective, we introduce and study right (resp., left) qnil-duo rings. We show that this concept is not left-right symmetric. Among others, it is proved that if the Hurwitz series ring $H(R ; \alpha)$ is right qnil-duo, then $R$ is right qnil-duo. Every right qnil-duo ring is abelian. A right qnil-duo exchange ring has stable range 1.

Key words and phrases: quasinilpotent element, duo ring, qnil-duo ring.
\end{abstract}

\footnotetext{
${ }^{1}$ Department of Mathematics, Hacettepe University, Ankara, Turkey

2 Department of Mathematics, Bilkent University, Ankara, Turkey

${ }^{3}$ Department of Mathematics, Ankara University, Ankara, Turkey

E-mail: harmanci@hacettepe.edu.tr(Harmanci A.), yosum@fen.bilkent.edu.tr(Kurtulmaz Y.), bungorescience.ankara.edu.tr(Ungor B.)
}

\section{Introduction}

Throughout this paper, all rings are associative with identity. Let $N(R), J(R), U(R), C(R)$ and $\operatorname{Id}(R)$ denote the set of all nilpotent elements, the Jacobson radical, the set of all invertible elements, the center and the set of all idempotents of a ring $R$, respectively. We denote the $n \times n$ full (resp., upper triangular) matrix ring over $R$ by $M_{n}(R)$ (resp., $\left.U_{n}(R)\right)$, and $D_{n}(R)$ stands for the subring of $U_{n}(R)$ consisting of all matrices which have equal diagonal entries and $V_{n}(R)=\left\{\left(a_{i j}\right) \in D_{n}(R) \mid a_{i j}=a_{(i+1)(j+1)}\right.$ for $i=1, \ldots, n-2$ and $\left.j=2, \ldots, n-1\right\}$ is a subring of $D_{n}(R)$. Let $\mathbb{Z}$ and $\mathbb{Z}_{n}$ denote the ring of integers and the ring of integers modulo $n$, where $n \geq 2$.

In [4], E.H. Feller introduced the notion of duo rings, that is, a ring is called right (resp., left) $d u o$ if every right (resp., left) ideal is an ideal, in other words, $R a \subseteq a R$ (resp., $a R \subseteq R a$ ) for every $a \in R$, and a ring is said to be $d u o$ if it is both right and left duo. The duo ring property was studied in different aspects. For example, in [5], the concept of right unit-duo ring was introduced, namely, a ring $R$ is called right unit-duo if for every $a \in R, U(R) a \subseteq a U(R)$. Left unit-duo rings are defined similarly. In [9], the normal property of elements on Jacobson and nil radicals were concerned. A ring $R$ is called right normal on Jacobson radical if $J(R) a \subseteq a J(R)$ for all $a \in R$. Left normal on Jacobson radical rings can be defined analogously. Also in [9], on the one hand, a ring $R$ is said to satisfy the right normal on upper nilradical if $N^{*}(R) a \subseteq$ $a N^{*}(R)$ for all $a \in R$, where $N^{*}(R)$ is the upper nilradical of $R$. Similarly, left normal on upper nilradical rings are defined similarly. On the other hand, a ring $R$ is said to satisfy the right normal on lower nilradical if $N_{*}(R) a \subseteq a N_{*}(R)$ for all $a \in R$, where $N_{*}(R)$ is the lower

$\mathrm{y} \Delta \mathrm{K} 512.554,512.743 .3$

2020 Mathematics Subject Classification: 16U80, 16U60, 16N40. 
nilradical of $R$. Similarly, left normal on lower nilradical rings are defined similarly. Also, a ring $R$ is called right nilpotent-duo if $N(R) a \subseteq a N(R)$ for every $a \in R$. Left nilpotent duo rings are defined similarly (see [7]).

Motivated by the works on duo property for rings, the goal of this paper is to approach the notion of duo rings by the way of quasinilpotent elements, in this regard, we introduce the notion of qnil-duo rings. Firstly, we investigate some properties of quasinilpotent elements, which we need for the investigation of qnil-duo property. Then we study some properties of this class of rings and observe that being a qnil-duo ring need not be left-right symmetric. It is proved that any right (resp., left) qnil-duo ring is abelian, and any exchange right (resp., left) qnil-duo ring has stable range 1. It is observed that regularity and strongly regularity coincide for right (resp., left) qnil-duo rings. We also study on some extensions of rings such as Dorroh extensions, Hurwitz series rings and some subrings of matrix rings in terms of qnil-duo property.

\section{Some properties of quasinilpotents}

Let $R$ be a ring and $a \in R$. The commutant and double commutant of $a$ in $R$ are defined by $\operatorname{comm}(a)=\{b \in R \mid a b=b a\}$ and $\operatorname{comm}^{2}(a)=\{b \in R \mid b c=c b$ for all $c \in \operatorname{comm}(a)\}$, respectively, and $R^{\text {qnil }}=\{a \in R \mid 1+a x$ is invertible in $R$ for every $x \in \operatorname{comm}(a)\}$. Elements of the set $R^{\text {qnil }}$ are called quasinilpotent (see [6]). Note that $J(R)=\{a \in R \mid 1+a x$ is invertible for every $x \in R\}$. If $a \in N(R)$ and $x \in \operatorname{comm}(a)$, then $a x \in N(R)$ and $1+a x \in U(R)$. So $J(R) \subseteq R^{\text {qnil }}, N(R) \subseteq R^{\text {qnil }}$ and $R^{\text {qnil }}$ does not contain invertible elements, $0 \in R^{\text {qnil }}$ but the identity is not in $R^{q n i l}$. In this section, we start to expose some properties of $R^{\text {qnil }}$ and continue to study some other properties of quasinilpotent elements in rings.

Example 1. There are rings $R$ such that $J(R)$ is strictly contained in $R^{\text {qnil }}$.

Proof. Let $F$ be a field and $R=M_{n}(F)$ for some positive integer $n$. Then $J(R)=0$ and the matrix unit $E_{1 n}$ belongs to $R^{\text {qnil }}$ but not $J(R)$.

We now mention some of the known facts about quasinilpotents for an easy reference.

Proposition 1. (1) Let $R$ be a ring, $n$ be a positive integer, and $a \in R$. If $a^{n} \in R^{\text {qnil }}$, then $a \in R^{\text {qnil }}$, in particular every nilpotent element is in $R^{\text {qnil }}$ ([3, Proposition 2.7]).

(2) If $R$ is a local ring, then $U(R) \cap R^{\text {qnil }}=\varnothing$ and $R=U(R) \cup R^{\text {qnil }}$ ([3, Theorem 3.2]).

(3) Let $R$ be a ring, $a, b \in R$. Then $a b \in R^{\text {qnil }}$ if and only if $b a \in R^{\text {qnil }}$ ([11, Lemma 2.2]).

(4) Let $a \in R^{\text {qnil }}$ and $r \in U(R)$. Then $r^{-1}$ ar $\in R^{\text {qnil }}$ ([3, Lemma 2.3]).

(5) Let $e \in \operatorname{Id}(R)$. Then $(e R e)^{\text {qnil }}=(e \operatorname{Re}) \cap R^{\text {qnil }}([16$, Lemma 3.5]).

In the following, we determine quasinilpotent elements in some classes of rings.

Lemma 1. Let $R$ be a ring. Then the following hold.

(1) $\left\{\left[\begin{array}{ll}a & b \\ 0 & c\end{array}\right] \mid a, c \in R^{q n i l}, b \in R\right\} \subseteq U_{2}(R)^{\text {qnil }}$.

(2) $\left\{\left[\begin{array}{ll}a & b \\ 0 & a\end{array}\right] \mid a \in R^{q n i l}, b \in R\right\} \subseteq D_{2}(R)^{\text {qnil }}$.

(3) Let $A=\left[\begin{array}{ll}a & b \\ 0 & a\end{array}\right] \in D_{2}(R)^{\text {gnil }}$ with $b \in \operatorname{comm}^{2}(a)$. Then $a \in R^{\text {qnil }}$. 
Proof. (1) Let $a, c \in R^{\text {qnil }}, b \in R, A=\left[\begin{array}{ll}a & b \\ 0 & c\end{array}\right] \in U_{2}(R)$ and $B=\left[\begin{array}{ll}x & y \\ 0 & z\end{array}\right] \in \operatorname{comm}(A)$. Then $A B=B A$ implies $1-a x$ and $1-c z$ are invertible. Hence $I_{2}-A B$ is invertible. So $A \in R^{q n i l}$.

(2) Let $A=\left[\begin{array}{ll}a & b \\ 0 & a\end{array}\right] \in D_{2}(R)$ with $a \in R^{q n i l}, b \in R$ and $B=\left[\begin{array}{ll}x & y \\ 0 & x\end{array}\right] \in \operatorname{comm}(A)$. Then $A B=B A$ implies $x \in \operatorname{comm}(a)$. Then $1-a x$ is invertible. Hence $I_{2}-A B$ is invertible. So $A \in D_{2}(R)^{\text {qnil }}$.

(3) Clear.

One may think of the following question.

Question 1. Are the reverse inclusions (1) and (2) in Lemma 1 true?

Proposition 2. Let $\left(R_{i}\right)_{i \in I}$ be a family of rings for some index set $I$ and let $R=\prod_{i \in I} R_{i}$. Then $R^{\text {qnil }}=\prod_{i \in I} R_{i}^{\text {qnil }}$.

Proof. Let $\left(a_{i}\right),\left(x_{i}\right) \in R$. Then $\left(x_{i}\right) \in \operatorname{comm}\left(a_{i}\right)$ if and only if $x_{i} \in \operatorname{comm}\left(a_{i}\right)$ for all $i \in I$. Hence $1+\left(a_{i}\right)\left(x_{i}\right)$ is invertible in $R$ if and only if $1+a_{i} x_{i}$ is invertible in $R_{i}$ for every $i \in I$. So the result follows.

Let $R$ be an algebra over a commutative ring $S$. The Dorroh extension (or ideal extension) of $R$ by $S$ denoted by $I(R, S)$ is the direct product $R \times S$ with usual addition and multiplication defined by $\left(a_{1}, b_{1}\right)\left(a_{2}, b_{2}\right)=\left(a_{1} a_{2}+b_{1} a_{2}+b_{2} a_{1}, b_{1} b_{2}\right)$ for $a_{1}, a_{2} \in R$ and $b_{1}, b_{2} \in S$.

Lemma 2. Let $I(R, S)$ be an ideal extension of $R$ by $S$. Then the following hold.

(1) For $(a, b) \in I(R, S),(c, d) \in \operatorname{comm}(a, b)$ if and only if $c \in \operatorname{comm}(a)$.

(2) $(a, b)$ has an inverse $(c, d)$ in $I(R, S)$ if and only if $(a+b)(c+d)=1=(c+d)(a+b)$ and $b d=d b=1$.

Proof. (1) $(c, d) \in \operatorname{comm}(a, b)$ if and only if $(a, b)(c, d)=(c, d)(a, b)$ if and only if $a c+d a+b c=$ $c a+d a+b c$ and $b d=d b$ if and only if $a c=c a$ and $b d=d b$ if and only if $c \in \operatorname{comm}(a)$.

(2) $(a, b)(c, d)=(0,1)=(c, d)(a, b)$ if and only if $a c+d a+b c=c a+d a+b c=0$ and $b d=$ $d b=1$ if and only if $a c+d a+b c+b d+(-b d)=(a+b)(c+d)-1=0$ and $b d=d b=1$.

Proposition 3. Let $I(R, S)$ be an ideal extension of $R$ by $S$. Then

(1) $(R, 0)^{\text {qnil }}=(R, 0) \cap I(R, S)^{\text {qnil }}$,

(2) $(0, S) \cap I(R, S)^{\text {qnil }} \subseteq(0, S)^{\text {qnil }}$.

Proof. (1) Let $(x, 0) \in(R, 0)^{\text {gnil }}$ and $(a, b) \in I(R, S)$ with $(a, b) \in \operatorname{comm}(x, 0)$. Then $a \in \operatorname{comm}(x)$ and so $1+x a$ is invertible in $R$. We prove $(0,1)+(x, 0)(a, b)$ is invertible. Since $S$ lies in the center of $R, a+b \in \operatorname{comm}(x)$. Hence $1+x(a+b)$ is invertible, say $(1+x(a+b))(u+1)=(u+1)(1+x(a+b))=1$. This implies that $u(x(a+b))+$ $x(a+b)+u=0$. Hence $((0,1)+(x, 0)(a, b))(u, 1)=(u, 1)((0,1)+(x, 0)(a, b))=(0,1)$ for all $(a, b) \in \operatorname{comm}(x, 0)$. So $(x, 0) \in I(R, S)^{\text {qnil }}$. Conversely, let $(x, 0) \in(R, 0) \cap I(R, S)^{\text {qnil }}$ and $(r, 0) \in \operatorname{comm}(x, 0)$. Hence, $(0,1)+(x, 0)(r, 0)=(r x, 1)$ is invertible. Let $(a, b)$ be the inverse of $(r x, 1)$. Then $(r x, 1)(a, b)=(0,1)$ implies $b=1$ and $r x a+r x+a=0 .(a, 1)(r x, 1)=(0,1)$ implies $a r x+a+r x=0$. Hence $(1+a)(1+r x)=1$ and $(1+r x)(1+a)=1$. Hence $(1,0)+(r, 0)(x, 0)$ is invertible in $(R, 0)$ for all $(r, 0) \in \operatorname{comm}(x, 0)$. Thus $(x, 0) \in(R, 0)^{\text {qnil }}$ or $(R, 0) \cap I(R, S)^{\text {qnil }} \subseteq(R, 0)^{\text {qnil }}$. 
(2) Let $(0, s) \in(0, S) \cap I(R, S)^{\text {qnil }}$. Let $(0, b) \in(0, S)$ with $(0, b) \in \operatorname{comm}(0, s)$. Then $(0,1)+(0, s)(0, b)=(0,1+s b)$ is invertible in $I(R, S)$. There exists $(u, v) \in I(R, S)$ such that $(0,1+s b)(u, v)=((1+s b) u,(1+s b) v)=(0,1)=(u, v)(0,1+s b)=((1+s b) u, v(1+s b))$. Hence $(1+s b) v=v(1+s b)=1$ and $(1+s b) u=0$. Hence $u=0$. Thus $(0,1)+(0, s)(0, b)=$ $(0,1+s b)$ is invertible in $(0, S)$ with inverse $(0, v) \in(0, S)$. It follows that $(0, s) \in(0, S)^{\text {gnil }}$ and so $(0, S) \cap I(R, S)^{\text {qnil }} \subseteq(0, S)^{\text {qnil }}$.

The following gives us necessary and sufficient conditions for $(0, S)^{\text {gnil }}$ to be contained in $I(R, S)^{\text {qnil }}$.

Theorem 1. Let $I(R, S)$ be the ideal extension of an algebra $R$ by a commutative ring $S$. Let $(0, i) \in(0, S)^{\text {gnil }}$. Then $(0, i) \in I(R, S)^{\text {qnil }}$ if and only if for every $(a, b) \in \operatorname{comm}(0, i)$ there exists $(u, v) \in I(R, S)$ such that $(i(a+b)+1)(u+v)=(1+i b) v=1$.

Proof. Assume that $(0, i) \in I(R, S)^{\text {qnil }}$. Let $(a, b) \in \operatorname{comm}(0, i)$ in $I(R, S)$. Then $(0,1)+$ $(0, i)(a, b)$ must be invertible. There exists $(u, v) \in I(R, S)$ such that $(0,1)=((0,1)+$ $(0, i)(a, b))(u, v)$. It follows that $(0,1)=((0,1)+(0, i)(a, b))(u, v)=(i a, 1+i b)(u, v)=$ $(i a u+(1+i b) u+v(i a),(1+i b) v)$. Then $i a u+(1+i b) u+i a v=0$ and $(1+i b) v=1$. They lead us $(i(a+b)+1)(u+v)=(1+i b) v$. Hence $(i(a+b)+1)(u+v)$ is invertible. Conversely, note that $i a u+(1+i b) u+i a v=0$ if and only if $(i(a+b)+1)(u+v)=(1+i b) v$. Assume that for $(0, i) \in(0, S)$ there exists $(u, v) \in I(R, S)$ such that $(i(a+b)+1)(u+v)=(1+i b) v=1$. Then by concealing paranthesis we may reach that $(0,1)=((0,1)+(0, i)(a, b))(u, v)$ for $(a, b) \in$ $\operatorname{comm}(0, i)$. Hence $(0, i) \in I(R, S)^{\text {qnil }}$.

Let $R$ be a ring and $S$ a subring of $R$ with the same identity as that of $R$ and

$$
T[R, S]=\left\{\left(r_{1}, r_{2}, \ldots, r_{n}, s, s, \ldots\right): r_{i} \in R, s \in S, n \geq 1,1 \leq i \leq n\right\} .
$$

Then $T[R, S]$ is a ring under the componentwise addition and multiplication. Note that $N(T[R, S])=T[N(R), N(S)]$ and $C(T[R, S])=T[C(R), C(R) \cap C(S)]$.

Proposition 4. Let $R$ be a ring and $S$ a subring of $R$ with the same identity as that of $R$.

(1) If $A=\left(a_{1}, a_{2}, a_{3}, \ldots, a_{n}, s, s, s, \ldots\right) \in T[R, S]^{\text {qnil }}$, then $a_{i} \in R^{\text {gnil }}$ for $i=1,2,3, \ldots, n$ and $s \in S^{\text {qnil }}$.

(2) If $a \in R^{\text {qnil }}$ and $s \in S^{\text {qnil }}$, then $A=(a, s, s, s, \ldots) \in T[R, S]^{\text {gnil }}$.

Proof. (1) Let $A=\left(a_{1}, a_{2}, \ldots, a_{n}, s, s, s, \ldots\right) \in T[R, s]^{\text {gnil }}$ and $b_{i} \in \operatorname{comm}\left(a_{i}\right)$ and $t \in \operatorname{comm}(s)$. Then $B=\left(b_{1}, b_{2}, \ldots, b_{n}, t, t, t, \ldots\right) \in \operatorname{comm}(A)$. Let $1=(1,1, \ldots, 1, \ldots)$ denote the identity of $T[R, S]$. So $1+A B$ is invertible. Therefore $1+a_{i} b_{i}$ is invertible for $i=1,2, \ldots, n$ and $1+s t$ is invertible in $S$. Hence $a_{i} \in R^{\text {qnil }}$ for $i=1,2, \ldots, n$ and $s \in S^{\text {qnil }}$.

(2) Let $a \in R^{\text {qnil }}, s \in S^{\text {qnil }}, A=(a, s, s, \ldots)$. If $B=\left(b_{1}, b_{2}, \ldots, b_{m}, t, t, t, \ldots\right) \in T[R, S]$ lies in $\operatorname{comm}(A)$, then $b_{1} \in \operatorname{comm}(a), b_{i} \in \operatorname{comm}(s)$ for $i=2,3, \ldots, m$ and $t \in \operatorname{comm}(s)$. Hence $1+a b_{1}$ and $1+s b_{i}$ are invertible in $R$, where $i=2,3, \ldots, m$ and $1+s t$ is invertible in $S$. Hence $1+A B$ is invertible in $T[R, S]$. So $A=(a, s, s, \ldots) \in T[R, S]^{\text {gnil }}$.

Let $R$ be a ring with an endomorphism $\alpha$ and let $H(R ; \alpha)$ be the set of formal expressions of the type $f(x)=\sum_{n=0}^{\infty} a_{n} x^{n}$, where $a_{n} \in R$ for all $n \geq 0$. Define addition as componentwise and $*$-product on $H(R ; \alpha)$ as follows: for $f(x)=\sum_{n=0}^{\infty} a_{n} x^{n}$ and $g(x)=\sum_{n=0}^{\infty} b_{n} x^{n}, f * g=$ $\sum_{n=0}^{\infty} c_{n} x^{n}$, where $c_{n}=\sum_{i=0}^{n}\left(\begin{array}{c}n \\ i\end{array}\right) a_{i} b_{n-i}$. Then $H(R ; \alpha)$ becomes a ring with identity containing 
$R$ under these two operations. The ring $H(R ; \alpha)$ is called the Hurwitz series ring over $R$. The Hurwitz polynomial ring $h(R ; \alpha)$ is the subring of $H(R ; \alpha)$ consisting of formal expressions of the form $\sum_{i=0}^{n}\left(\begin{array}{c}n \\ i\end{array}\right) a_{i} x^{i}$. Let $\epsilon: H(R ; \alpha) \rightarrow R$ defined by $\epsilon(f(x))=a_{0}$. Then $\epsilon$ is a homomorphism with $\operatorname{ker}(\epsilon)=x H(R ; \alpha)$ and $H(R ; \alpha) / \operatorname{ker}(\epsilon) \cong R$. There exist one-to-one correspondences between $H(R ; \alpha)$ and $R$ relating to invertible elements, commutants and ideals. Let $R[[x ; \alpha]]$ be the skew formal power series ring over $R$. The sum is the same but multiplication in $H(R, \alpha)$ is similar to the usual multiplication of $R[[x ; \alpha]]$, except that binomial coefficients appear in each term in the multiplication defined in $H(R, \alpha)$. Also, there is a ring homomorphism $\epsilon$ between $R[[x ; \alpha]]$ and $R$, defined by $\epsilon(f(x))=a_{0}$, where $f(x)=a_{0}+a_{1} x+a_{2} x^{2}+\cdots \in R[[x ; \alpha]]$. Clearly, $\epsilon$ is an onto map and $R[[x ; \alpha]] / \operatorname{ker}(\epsilon) \cong R$.

Lemma 3. Let $R$ be a ring and $\alpha$ a ring endomorphism of $R$. Then

(1) $U(H(R ; \alpha))=\epsilon^{-1} U(R)$,

(2) $U(R[[x ; \alpha]])=\epsilon^{-1} U(R)$.

Proof. It is routine.

In the next result, we determine the quasinilpotent elements of $H(R ; \alpha)$ and $R[[x ; \alpha]]$.

Proposition 5. (1) Let $H(R ; \alpha)$ be a skew Hurwitz series ring over $R$. Then

$$
H(R ; \alpha)^{q n i l}=\epsilon^{-1} R^{q n i l} .
$$

(2) Let $R[[x ; \alpha]]$ be a skew formal power series ring over $R$. Then $R([[x ; \alpha]])^{\text {gnil }}=\epsilon^{-1} R^{\text {qnil }}$.

Proof. (1) Let $f(x)=a_{0}+a_{1} x+a_{2} x^{2}+\cdots \in H(R ; \alpha)^{\text {gnil }}$ and $r \in R$ with $r \in \operatorname{comm}_{R}\left(a_{0}\right)$. Then $r \in \operatorname{comm}_{H(R ; \alpha)}\left(a_{0}\right)$. Then $1+f(x) r \in U(H(R ; \alpha))$. Hence $1+a_{0} r \in U(R)$. So $a_{0} \in R^{q n i l}$. Since $a_{0}=\epsilon(f(x)), f(x) \in \epsilon^{-1}\left(R^{\text {qnil }}\right)$. Conversely, let $g(x)=b_{0}+b_{1} x+b_{2} x^{2}+\cdots \in \epsilon^{-1}\left(R^{q n i l}\right)$. Then $\epsilon(g(x))=b_{0} \in R^{\text {gnil }}$. Let $h(x)=c_{0}+c_{1} x+c_{2} x^{2}+\cdots \in \operatorname{comm}_{H(R, \alpha)}(g(x))$. Then $c_{0} \in$ $\operatorname{comm}_{R}\left(b_{0}\right)$ and so $1+b_{0} c_{0} \in U(R)$. So $1+g(x) h(x) \in U(H(R, \alpha))$. Hence $g(x) \in H(R, \alpha)^{\text {qnil }}$. This completes the proof.

(2) Similar that of (1).

\section{Qnil-duo rings}

In this section, we deal with the right duo property on the set of quasinilpotent elements. By this means we give a generalization of commutativity from the perspective of quasinilpotents.

Definition 1. A ring $R$ is called right qnil-duo if $R^{\text {qnil }} a \subseteq a R^{\text {qnil }}$ for every $a \in R$. Similarly, $R$ is called left qnil-duo if $a R^{\text {qnil }} \subseteq R^{\text {qnil }} a$ for every $a \in R$. If $R$ is both right and left qnil-duo, then it is called qnil-duo, i.e. $R^{\text {qnil }} a=a R^{\text {qnil }}$ for every $a \in R$.

The qnil-duo property of rings is not left-right symmetric as the following example shows.

Example 2. Let $S=F(t)$ denote the quotient field of the polynomial ring $F[t]$ over a field $F$ and $\alpha: S \rightarrow S$ defined by $\alpha(f(t) / g(t))=f\left(t^{2}\right) / g\left(t^{2}\right)$. Let $R=S[[x ; \alpha]]$ denote the skew power series ring with $x a=\alpha(a) x$ for $a \in S$. Every element of $R$ is of the form $a=\sum_{i=0}^{\infty} a_{i} x^{i}$. For any $r=a_{0}+\sum_{i=1}^{\infty} a_{i} x^{i}$ with $a_{0} \neq 0$ is invertible. Hence $R^{q n i l}=x R$. 
This ring is considered in [2, Lemma 1.3 (3)], [8, Example 1] and in [7, Example 1.5]. As in the proof of [8, Example 1], for $t x^{m} \in t R^{\text {qnil }}$, there is no $g(x) \in R^{\text {qnil }}$ such that $t x^{m}=$ $g(x)$ t. Hence $R$ is not left qnil-duo. We show that $R$ is right qnil-duo. Let $f(x) \in R^{\text {qnil }}$, $g(x) \in R$. We show that there exists $f_{1}(x) \in R^{\text {qnil }}$ such that $f(x) g(x)=g(x) f_{1}(x)$. Assume that $g(x)$ is invertible. Then $f(x) g(x)=g(x)\left(g(x)^{-1} f(x) g(x)\right) \in g(x) R^{q n i l}$, otherwise, let $g(x)=h(x) x^{m}$, where $h(x)=a_{0}+a_{1} x^{+} a_{2} x^{2}+\cdots$ is invertible. Then

$$
\begin{aligned}
f(x) g(x) & =f(x) h(x) x^{m}=f(x) x^{m} h_{1}(x)=x^{m} f_{1}(x) h_{1}(x) \\
& =x^{m} h_{1}(x)\left(h_{1}(x)^{-1} f_{1}(x) h_{1}(x)\right)=g(x)\left(h_{1}(x)^{-1} f_{1}(x) h_{1}(x)\right) \in g(x) R^{q n i l}
\end{aligned}
$$

since $f(x)$ is not invertible and $f_{1}(x)$ is an application of $x^{m}$ to $f(x)$ from the right, therefore $f_{1}(x)=x^{k} f_{2}(x) \in R^{\text {qnil }}$ for some $k \geq 1$, by Proposition $1(4), h_{1}(x)^{-1} f_{1}(x) h_{1}(x) \in R^{q n i l}$. Thus $R$ is right qnil-duo.

Example 3. (1) All commutative rings, all division rings are qnil-duo.

(2) There are local rings that are not right qnil-duo.

Proof. (1) When $R$ is a commutative ring, it is both right and left qnil-duo. If $R$ is a division ring, then $R^{\text {qnil }}=\{0\}$, therefore $R$ is both right and left qnil-duo.

(2) Let $A=\mathbb{Z}_{4}[x, y]$ be the polynomial ring with non-commuting indeterminates $x$ and $y$ and $I$ be the ideal generated by the set $\left\{x^{3}, y^{2}, y x, x^{2}-x y, x^{2}-2,2 x, 2 y\right\}$. Consider the ring $R=A / I$. By [15, Example 7], $R$ is a local ring. It is easily checked that

$$
R^{q n i l}=\{0,2, x, y, 2+x, 2+y, 2+x+y, x+y\} \quad \text { and } \quad\left(R^{q n i l}\right)^{2} \neq 0,
$$

$2+x$ belongs to $R^{q n i l}$ since it is nilpotent, for $x \in R^{q n i l}$ and $y \in R, x y \in R^{q n i l} y$. It is easily checked that there is no $t \in R^{\text {qnil }}$ such that $x y=y t \in y R^{\text {qnil }}$. Hence $R$ is not right qnil-duo.

Lemma 4. Let $R$ be a ring with $R^{\text {qnil }}$ central in $R$. Then $R$ is qnil-duo.

Proof. Assume that $R^{q n i l}$ is central in $R$. Let $a \in R$ and $b \in R^{q n i l}$. Then $b$ being central implies $a b=b a \in a R^{q n i l}$.

Theorem 2. Let $\left\{R_{i}\right\}_{i \in I}$ be a family of rings for some index set $I$ and $R=\prod_{i \in I} R_{i}$. Then $R_{i}$ is right (resp., left) qnil-duo for each $i \in I$ if and only if $R$ is right (resp., left) qnil-duo.

Proof. Assume that $R_{i}$ is right (resp., left) qnil-duo for each $i \in I$. Let $a=\left(a_{i}\right) \in R, b=\left(b_{i}\right) \in$ $R^{\text {qnil }}$. By Proposition $2, b_{i} \in R_{i}^{\text {qnil }}$ for each $i \in I$. By assumption there exists $c_{i} \in R_{i}^{\text {qnil }}$ such that $b_{i} a_{i}=a_{i} c_{i}$ for each $i \in I$. Set $c=\left(c_{i}\right)$. Then $b a=a c \in a R^{q n i l}$. Hence $R^{q n i l} a \subseteq a R^{\text {qnil }}$. Conversely, suppose that $R$ is right qnil-duo. Let $a_{i} \in R_{i}$ and $b_{i} \in R_{i}^{\text {qnil }}$, where $i \in I$. Let $a=\left(a_{i}\right), b=\left(b_{i}\right) \in R$, where $i^{t h}$-entry of $a$ is $a_{i}$ and the other entries are 0 and $i^{\text {th }}$-entry of $b$ is $b_{i}$ and the other entries are 0 , respectively. Then $a=\left(a_{i}\right) \in R$ and by Proposition $2, b \in R^{\text {qnil }}$. The supposition implies there exists $c=\left(c_{i}\right) \in R^{q n i l}$ such that $b a=a c$. Comparing entries of both sides we have $b_{i} a_{i}=a_{i} c_{i}$. By Proposition $2, c_{i} \in R_{i}^{q n i l}$. Thus for each $i \in I, R_{i}$ is right qnil-duo. Similarly, it is proven that for each $i \in I, R_{i}$ is left qnil-duo.

Recall that a ring $R$ is called abelian if every idempotent in $R$ is central. 
Theorem 3. Let $R$ be a ring. Then the following hold.

(1) $e x-e x e$ and $x e-e x e \in R^{\text {qnil }}$ for every $x$ and $e^{2}=e \in R$.

(2) Right (resp., left) qnil-duo rings are abelian.

(3) Let $R$ be a ring and $e \in I d(R)$. If $R$ is a right (resp., left) qnil-duo ring, then $e R$ and $(1-e) R$ are right (resp., left) qnil-duo rings. The converse holds if $e$ is central.

Proof. (1) Let $t \in \operatorname{comm}(e x-e x e)$. Then $t(e x-e x e)=(e x-e x e) t$. So we have $(t(x e-e x e))^{2}=0$. Hence $1-(e x-e x e) t$ is invertible and so $e x-e x e \in R^{\text {qnil }}$. Similarly, $x e-e x e \in R^{\text {qnil }}$.

(2) Let $e^{2}=e \in R$. By hypothesis, $R^{\text {qnil }} e \subseteq e R^{\text {qnil }}$. By (1), $x e-e x e \in R^{\text {qnil }}$ for all $x \in R$. It implies for any $x \in R$, there exists $t \in R^{\text {qnil }}$ such that $(x e-e x e) e=e t$. Multiplying the latter equality by $e$ from the left we have $e t=0$. So, $x e=e x e$. Similarly, $e x=$ exe since ex - exe $\in R^{\text {qnil }}$ by (1). Hence $R$ is abelian.

(3) It is clear by Theorem 2.

Corollary 1. Let $R$ be a right (resp., left) qnil-duo ring and $e \in \operatorname{Id}(R)$. Then the corner ring $e$ Re is a right (resp., left) qnil-duo ring.

Proof. The ring $R$ being right (resp., left) qnil-duo implies that $e$ is central in $R$ by Theorem 3 (2). Hence Theorem 3 (3) completes the proof.

Theorem 4. Every right (resp., left) qnil-duo ring is directly finite.

Proof. Let $R$ be a right qnil-duo ring and $a, b \in R$ with $a b=1$. Set $e=1-b a$. Then $e$ is an idempotent. By Theorem $3, e$ is central. So, $0=a e=e a$. Hence $0=a-b a^{2}$. Multiplying the latter by $b$ from the right, we get $1=b a$.

There is a directly finite ring that is neither right nor left qnil-duo.

Example 4. Consider the ring $R=M_{2}\left(\mathbb{Z}_{2}\right)$. Then $R$ is a directly finite ring but not abelian. Hence it is neither right nor left qnil-duo.

We apply Theorem 3 to show that full matrix rings and upper triangular matrix rings need not be right (resp., left) qnil-duo. But there are some subrings of full matrix rings that are qnil-duo.

Example 5. (1) For any ring $R$ and any positive integer $n, M_{n}(R)$ and $U_{n}(R)$ are neither right nor left qnil-duo.

(2) If $R$ is commutative, then $V_{n}(R)$ is qnil-duo.

(3) $V_{n}(R[[x ; \sigma]])$ is neither right nor left qnil-duo.

Proof. (1) The rings $M_{n}(R)$ and $U_{n}(R)$ are not abelian. By Theorem 3 (2), they are neither right nor left qnil-duo.

(2) If $R$ is a commutative ring, $V_{n}(R)$ is also commutative, therefore it is right and left qnil-duo.

(3) Let $R$ be a ring with an endomorphism $\sigma$. Assume that there exists $a_{1} \in R$ such that $\sigma\left(a_{1}\right) \notin a_{1} R$. Let $A=\left[\begin{array}{ccc}x & x & x \\ 0 & x & x \\ 0 & 0 & x\end{array}\right] \in V_{3}(R[[x ; \sigma]])^{\text {gnil }}, B=\left[\begin{array}{ccc}a_{1} & a_{2} & a_{3} \\ 0 & a_{1} & a_{2} \\ 0 & 0 & a_{1}\end{array}\right] \in V_{3}(R[[x ; \sigma]])$. Assume that there exists $D \in V_{3}(R[[x ; \sigma]])^{\text {qnil }}$ such that $A B=B D$. Then $(1,1)$ entry of $A B$ is 
$\sigma\left(a_{1}\right) x$ and that of $B D$ is $a_{1} x f(x)$ for some $f(x) \in R[[x ; \sigma]]$. This contradicts the choice of $\sigma$ and $a_{1}$. Therefore $V_{3}(R[[x ; \sigma]])$ is not right qnil-duo. Similarly, it can be shown that $V_{3}(R[[x ; \sigma]])$ is not left qnil-duo.

Theorem 5. Let $R$ be a local ring with $\left(R^{q n i l}\right)^{2}=0$. Then $R$ is right (resp., left) qnil-duo.

Proof. By Proposition 1 (2), we have $R=U(R) \cup R^{\text {qnil }}$. We prove $R^{\text {qnil }} a \subseteq$ a $R^{\text {qil }}$. Let $a \in R$, $b \in R^{q n i l}$. If $b a=0$, then we are done since $b a=0=a 0 \in a R^{\text {qnil }}$. Otherwise, i.e. if $b a \neq 0$, then we divide the proof in some cases.

Case I. Let $a \notin R^{\text {qnil }}$. Then $a \in U(R)$. By Proposition 1 (4), $a^{-1} b a \in R^{\text {qnil }}$ since $b \in R^{q n i l}$. Then $b a=a\left(a^{-1} b a\right) \in a R^{q n i l}$.

Case II. Let $a \in R^{q n i l}$. By hypothesis, $b a=0$, this contradicts with $b a \neq 0$.

Therefore $R$ is a right qnil-duo ring. Similarly, we may prove $a R^{q n i l} \subseteq R^{\text {qnil }} a$ for each $a \in R$.

As an illustration of Theorem 5, we give the following examples. Also, the condition $\left(R^{\text {qnil }}\right)^{2}=0$ in Theorem 5 is not superfluous.

Example 6. (1) Consider the ring $R=\left\{\left[\begin{array}{lll}a & b & c \\ 0 & a & 0 \\ 0 & 0 & a\end{array}\right] \in D_{3}\left(\mathbb{Z}_{4}\right)\right\}$. Then

$$
R^{\text {qnil }}=\left\{\left[\begin{array}{lll}
a & b & c \\
0 & a & 0 \\
0 & 0 & a
\end{array}\right] \in R \mid a \in 2 \mathbb{Z}_{4}, b, c \in \mathbb{Z}_{4}\right\} .
$$

So, $\left(R^{\text {qnil }}\right)^{2}=0$. By Theorem 5, $R$ is qnil-duo.

(2) Let $R$ denote the ring in Example 3. Then $R^{\text {qnil }}=\{0,2, x, y, 2+x, 2+y, 2+x+y, x+y\}$ and $\left(R^{\text {qnil }}\right)^{2} \neq 0$ and $2+x$ belongs to $R^{\text {qnil }}$ since it is nilpotent and $(2+x)^{2} \neq 0$. Since $R$ is local and $R^{\text {qnil }}$ does not contain invertible elements, $R^{\text {qnil }}=J(R)$. To complete the proof we may assume that $x, y \in R^{q n i l}$. Then $x y=2$ and $x y \in R^{q n i l} y$. It is easily checked that there is no $t \in R^{\text {qnil }}$ such that $x y=y t \in y R^{\text {qnil }}$. Hence $R$ is not right qnil-duo. Compare to Theorem 5 .

Note that by Theorem 5 , if $R$ is a division ring, $D_{2}(R)$ is a qnil-duo ring. One may ask whether $D_{2}(R)$ is qnil-duo over a domain $R$. The following example answers negatively.

Example 7. Consider the ring $D_{2}(R[[x]])$ in $\left[9\right.$, Example 1.4 (1)]. It is proved that $D_{2}(R[[x]])$ is neither right nor left normal property of elements on Jacobson radical. Since $J\left(D_{2}(R[[x]])\right)=$ $D_{2}(R[[x]])^{\text {gnil }}, D_{2}(R[[x]])$ is neither right nor left qnil-duo.

Theorem 6. Let $R$ be a domain. If $D_{2}(R)$ is right (resp., left) qnil-duo, then $R$ is right (resp., left) qnil-duo.

Proof. Assume that $D_{2}(R)$ is right qnil-duo. Let $a \in R$ and $b \in R^{\text {qnil }}$. Consider $A=\left[\begin{array}{ll}a & 0 \\ 0 & a\end{array}\right] \neq 0$, $B=\left[\begin{array}{ll}b & 0 \\ 0 & b\end{array}\right] \neq 0$. Let $X=\left[\begin{array}{ll}x & y \\ 0 & x\end{array}\right] \in \operatorname{comm}(B)$. Then $I_{2}-B X$ is invertible since $1-b x$ is invertible in $R$. Hence $B A \in D_{2}(R)^{\text {qnil }} A$. There exists $C=\left[\begin{array}{ll}c & d \\ 0 & c\end{array}\right] \in D_{2}(R)^{\text {qnil }}$ such that $B A=A C$. Then $b a=a c$ and $a d=0$. By hypothesis $d=0$. By Lemma 1(3), $c \in R^{q n i l}$. It follows that $b a=a c \in a R^{q n i l}$. Hence $R^{q n i l} a \subseteq a R^{q n i l}$. 
Recall that a ring $R$ is said to have stable range 1 if for any $a, b \in R$ satisfying $a R+b R=R$, there exists $y \in R$ such that $a+b y$ is right invertible (cf. [14]). In [12], a ring $R$ is called exchange if for any $x \in R$, there exists $e \in \operatorname{Id}(R)$ such that $e \in R x$ and $1-e \in R(1-x)$, and it is proved that for an abelian ring $R, R$ is exchange if and only if it is clean, and $R$ is exchange if and only if idempotents lift modulo every left (or right) ideal.

Theorem 7. The following hold.

(1) Right (resp., left) qnil-duo exchange rings have stable range 1.

(2) Right (resp., left) qnil-duo regular rings (in the sense of von Neumann) are strongly regular.

Proof. (1) Let $R$ be a right qnil-duo exchange ring. By Theorem $3, R$ is abelian. Hence [17, Theorem 6] implies $R$ has stable range 1 .

(2) Let $R$ be a qnil-duo regular ring and $a \in R$. There exists $b \in R$ such that $a=a b a$. Then $a b=(a b)^{2}, b a=(b a)^{2} \in \operatorname{Id}(R)$. By Theorem 3, $a b$ is central. So, $a=a b a=a^{2} b$. Hence $R$ is strongly regular.

Let $R$ be a ring. The Jacobson radical of the polynomial ring $R[x]$ is $J(R[x])=N[x]$, where $N=J(R[x]) \cap R$ is a nil ideal of $R$. Then $N \subseteq R^{\text {qnil }}$ and $J(R[[x]])=x R[[x]]$. Therefore, $R[[x]]^{\text {qnil }}=x R[[x]]$. One may wonder whether or not $R[x]$ and $R[[x]]$ are qnil-duo. The following example shows that $R[x]$ and $R[[x]]$ need not be right qnil-duo.

Example 8. (1) Let $F$ be a field, $R=M_{n}(F)$ and consider the ring $R[x]$. Observe that $M_{n}(F[x])$ is not right (or left) qnil-duo for any positive integer $n \geq 2$ by Example 5. It follows that $R[x]$ is not right (or left) qnil-duo since $M_{n}(F)[x] \cong M_{n}(F[x])$.

(2) Let $R=A /(a b-b a-1)$ denotes the Weyl algebra discussed in [9, Example 1.2 (2)]. Let $S=R[[x]]$. Then $S^{q n i l}=x R[[x]]=J(R[[x]]), R$ is a domain and $R[[x]]$ is abelian. It is proved that $R[[x]]$ is neither right normal nor left normal on $J(R)$. Therefore, $R[[x]]$ is neither right qnil-duo nor left qnil-duo.

Question 2. Under what conditions are the rings $R[x]$ and $R[[x]]$ right qnil-duo?

Theorem 8. Let $R$ be an algebra over a commutative ring $S$. Consider the Dorroh extension (or ideal extension) $I(R, S)$ of $R$ by $S$. If $I(R, S)$ is right qnil-duo, then so is $R$.

Proof. Assume that $I(R, S)$ is right qnil-duo. Let $a \in R, b \in R^{q n i l}$. Then $(a, 0) \in I(R, S)$ and $(b, 0) \in I(R, S)^{\text {qnil }}$. Indeed, let $(x, y) \in \operatorname{comm}(b, 0)$. By Lemma $2, x \in \operatorname{comm}(b)$. Since $R$ is an algebra over $S$, we have $x+y \in \operatorname{comm}(b)$. Then $1+b(x+y)$ is invertible in $R$ with inverse $t$. Again by Lemma $2,(0,1)+(b, 0)(x, y)$ is invertible in $I(R, S)$ with the inverse $(t-1,1)$. Then $(b, 0)(a, 0) \in I(R, S)^{\text {qnil }}(a, 0)$. There exists $(c, s) \in I(R, S)^{\text {qnil }}$ such that $(b, 0)(a, 0)=(a, 0)(c, s)$. So, $b a=a(c+s)$.

To complete the proof we show $c+s \in R^{q n i l}$. Let $x \in \operatorname{comm}(c+s)$. Then $c x+s x=s c+x s$. Since $R$ is an algebra over $S, s x=x s$, this implies $c x=x c$, and so $x \in \operatorname{comm}(c)$. Hence $(x, 0) \in \operatorname{comm}(c, s)$. Since $(c, s) \in I(R, S)^{\text {qnil }},(0,1)+(c, s)(x, 0)$ is invertible in $I(R, S)$. Thus $1+(c+s) x$ is invertible in $R$ by Lemma 2(2). So, $c+s \in R^{q n i l}$. Therefore $R$ is right qnilduo. 
Proposition 6. Let $R$ be a ring and $S$ a subring of $R$. If $T[R, S]$ is right qnil-duo, then so are $R$ and $S$. The converse holds if $S^{\text {qnil }} \subseteq R^{\text {qnil }}$.

Proof. Assume that $T[R, S]$ is right qnil-duo. Let $a \in R, b \in R^{\text {qnil }}$. Let $A=(a, 0,0,0, \ldots)$, $B=(b, 0,0,0, \ldots) \in T[R, S]$. By Proposition $4, B \in T[R, S]^{\text {qnil }}$. By supposition there exists $C=$ $\left(c_{1}, c_{2}, \cdots, c_{m}, t, t, \ldots\right) \in T[R, S]^{\text {qnil }}$ such that $B A=A C$. Hence $b a=a c_{1}$. By Proposition 4, $c_{1} \in R^{q n i l}$. Similarly, let $s \in S, t \in S^{\text {qnil }}$ and $C=(0, s, s, s, \ldots), D=(0, t, t, t, \ldots) \in T[R, S]$. By Proposition $4, D \in T[R, S]^{\text {gil }}$. There exists $D^{\prime}=\left(d_{1}, d_{2}, d_{3}, \ldots, d_{l}, u, u, u, \ldots\right) \in T[R, S]^{\text {qnil }}$ such that $D C=C D^{\prime}$. By Proposition $4, u \in S^{q n i l}$ and $t s=s u \in s S^{q n i l}$.

Suppose that $R$ and $S$ are right qnil-duo and $S^{\text {qnil }} \subseteq R^{\text {qnil }}$. Let $A \in T[R, S], B \in T[R, S]^{\text {qnil }}$, where $A=\left(a_{1}, a_{2}, \ldots, a_{n}, s, s, \ldots\right), B=\left(b_{1}, b_{2}, \ldots, b_{m}, t, t, \ldots\right)$, we prove $B A=A C$ for some $C \in T[R, S]^{\text {qnil }}$. By Proposition $4, b_{i} \in R^{\text {qnil }}$ for $i=1,2, \ldots, m$ and $t \in S^{\text {qnil }}$. By supposition $b_{i} \in R^{\text {qnil }}$ implies $b_{i} a_{i}=a_{i} c_{i}$ for some $c_{i} \in R^{\text {qnil }}$. We divide the proof in some cases.

Case I. $n \leq m$. Then $b_{i} a_{i} \in R^{\text {qnil }} a_{i}$. Since $R$ is right qnil-duo, there exist $c_{i} \in R^{\text {qnil }}$ such that $b_{i} a_{i}=a_{i} c_{i}$ for each $1 \leq i \leq n$. For $n+1 \leq i \leq m, b_{i} s \in R^{\text {qnil }}$. There exist $c_{i} \in R^{\text {qnil }}$ such that $b_{i} s=s c_{i}$. For $t s \in S^{\text {qnil }} s$, there exists $l \in S^{\text {qnil }}$ such that $t s=s l \in s S^{\text {qnil }}$. Let $C=$ $\left(c_{1}, c_{2}, \ldots, c_{m}, l, l, l, \ldots\right)$. By Proposition $4(2), C \in T[R, S]^{\text {gnil }}$. Then $B A=A C \in A T[R, S]^{\text {gnil }}$.

Case II. $n>m$. Let $1 \leq i \leq m$. Then $b_{i} a_{i} \in R^{\text {qnil }} a_{i}$ and since $R$ is right qnil-duo, there exist $c_{i} \in R^{\text {qnil }}$ such that $b_{i} a_{i}=a_{i} c_{i}$. For $m+1 \leq i \leq n, t a_{i} \in S^{\text {qnil }} a_{i}$. By $S^{\text {qnil }} \subseteq R^{\text {qnil }}$, we have $t a_{i}=a_{i} c_{i} \in a_{i} R^{q n i l}$ for some $c_{i} \in R^{\text {qnil }}$. For $t s \in S^{q n i l}$, by supposition, there exists $l \in S^{\text {qnil }}$ such that $t s=s l \in s S^{\text {qnil }}$. Let $C=\left(c_{1}, c_{2}, \ldots, c_{n}, l, l, l, \ldots\right)$. By Proposition $4(2), C \in T[R, S]^{\text {gnil }}$. Then $B A=A C$. Hence $T[R, S]^{\text {gnil }} A \subseteq A T[R, S]^{\text {gnil }}$. It completes the proof.

Theorem 9. (1) Let $H(R ; \alpha)$ be a skew Hurwitz series ring over a ring $R$. If $H(R ; \alpha)$ is right qnil-duo, then $R$ is right qnil-duo.

(2) Let $R[[x ; \alpha]]$ be a skew formal power series ring over a ring $R$. If $R[[x ; \alpha]]$ is right qnil-duo, then $R$ is right qnil-duo.

Proof. (1) Suppose that $H(R ; \alpha)$ is a right qnil-duo ring. Let $a \in R^{\text {qnil }}$ and $b \in R$. By the definition of $\epsilon$ and Proposition 5, there exist $f(x), g(x) \in H(R ; \alpha)$ with $f(x) \in H(R ; \alpha)^{\text {qnil }}$ and $\epsilon(f(x))=a, \epsilon(g(x))=b$. There exists $h(x)=c_{0}+c_{1} x+c_{2} x^{2}+\cdots \in H(R ; \alpha)^{q n i l}$ such that $f(x) g(x)=g(x) h(x)$. Hence $\epsilon(f(x) g(x))=\epsilon(g(x) h(x))$ implies $a b=b c_{0} \in b R^{q n i l}$. Thus $R^{q n i l} b \subseteq b R^{\text {qnil }}$. The proof of (2) is similar to that of (1).

Note that by Proposition 5, we have the following equalities

$$
H(R ; \alpha)^{q n i l}=\epsilon^{-1} R^{q n i l} \quad \text { and } \quad R([[x ; \alpha]])^{q n i l}=\epsilon^{-1} R^{q n i l} .
$$

Here we raise the following two questions.

Question 3. By using the preceding equalities, one can prove the inverse statements in Theorem 9 (1) and (2) as: if $R$ is right qnil-duo, then

(1) is $H(R ; \alpha)$ right qnil-duo?

(2) is $R[[x ; \alpha]]$ right qnil-duo?

\section{Some subrings of matrix rings}

Besides, for any ring $R$ and any positive integer $n \geq 2, M_{n}(R)$ is not right (or left) qnil-duo, in this section, quasinilpotent elements of some subrings of full matrix rings are determined for the purpose of the use whether or not their subrings to be right (or left) qnil-duo. 
The rings $L_{(s, t)}(R)$. Let $R$ be a ring and $s, t \in C(R)$.

Let $L_{(s, t)}(R)=\left\{\left[\begin{array}{ccc}a & 0 & 0 \\ s c & d & t e \\ 0 & 0 & f\end{array}\right] \in M_{3}(R) \mid a, c, d, e, f \in R\right\}$, where the operations are defined as those in $M_{3}(R)$. Then $L_{(s, t)}(R)$ is a subring of $M_{3}(R)$.

Lemma 5. Let $A=\left[\begin{array}{ccc}a & 0 & 0 \\ s c & d & t e \\ 0 & 0 & f\end{array}\right] \in L_{(s, t)}(R)$. Then the following hold.

(1) $A$ is invertible in $L_{(s, t)}(R)$ if and only if $a, d$ and $f$ are invertible in $R$.

(2) If $a, d, f \in R^{q n i l}$, then $A \in L_{(s, t)}(R)^{\text {qnil }}$.

Proof. (1) One way is clear. Let $A=\left[\begin{array}{ccc}a & 0 & 0 \\ s c & d & t e \\ 0 & 0 & f\end{array}\right] \in L_{(s, t)}(R)$. Assume that $a, d$ and $f$ are invertible with $a x=x a=1, d z=z d=1$ and $f v=v f=1$, where $x, z, v \in R$. Consider $B=\left[\begin{array}{ccc}x & 0 & 0 \\ s y & z & t u \\ 0 & 0 & v\end{array}\right] \in L_{(s, t)}(R)$, where $y=-z c x$ and $u=-z e v$. Then $A B=B A=I_{3}$.

(2) Assume that $a, d, f \in R^{q n i l}$. We prove that $A \in L_{(s, t)}(R)^{q n i l}$. Let $B=\left[\begin{array}{ccc}x & 0 & 0 \\ s y & z & t u \\ 0 & 0 & v\end{array}\right] \in L_{(s, t)}(R)$ with $B \in \operatorname{comm}(A)$. It is easily checked that $x \in \operatorname{comm}(a), z \in \operatorname{comm}(d), v \in \operatorname{comm}(f)$. Then $1+a x, 1+d z, 1+f v$ are invertible in $R$. By (1), $I_{3}+A B=\left[\begin{array}{ccc}1+a x & 0 & 0 \\ s c x+s d y & 1+d z & t d u+t e v \\ 0 & 0 & 1+f v\end{array}\right]$ is invertible. So $A \in L_{(s, t)}(R)^{\text {qnil }}$.

Lemma 6. Let $A=\left[\begin{array}{ccc}a & 0 & 0 \\ s c & d & t e \\ 0 & 0 & f\end{array}\right] \in L_{(s, t)}(R)$. Then the following hold.

(1) If $A \in L_{(0, t)}(R)^{\text {gnil }}$, then $a \in R^{\text {qnil }}$.

(2) If $A \in L_{(s, 0)}(R)^{\text {qnil }}$, then $f \in R^{\text {qnil }}$.

(3) $A \in L_{(0,0)}(R)^{\text {qnil }}$ if and only if $a, d, f \in R^{\text {qnil }}$.

Proof. (1) Let $A=\left[\begin{array}{ccc}a & 0 & 0 \\ 0 & d & t e \\ 0 & 0 & f\end{array}\right] \in L_{(0, t)}(R)^{\text {gnil }}$ and $x \in \operatorname{comm}(a)$. Consider $B=\left[\begin{array}{ccc}x & 0 & 0 \\ 0 & 0 & 0 \\ 0 & 0 & 0\end{array}\right] \in$ $L_{(0, t)}(R)$. Then $B \in \operatorname{comm}(A)$. Since $A \in L_{(0, t)}(R)^{\text {qnil }}, I+A B$ is invertible in $L_{(0, t)}(R)$. By Lemma 5 (1), $1+a x \in U(R)$. Therefore $a \in R^{\text {qnil }}$.

(2) Similar to the proof of (1).

(3) The sufficiency follows from Lemma 5 (2). For the necessity, $a, f \in R^{\text {qnil }}$ by (1) and (2), respectively. Also, by the similar discussion in (1), we obtain $d \in R^{\text {qnil }}$. 
Theorem 10. Let $R$ be a ring. If $L_{(0, t)}(R)$ is right qnil-duo, then $R$ is a right qnil-duo ring.

Proof. Assume that $L_{(0, t)}(R)$ is right qnil-duo and let $a \in R$ and $b \in R^{q n i l}$. Consider $A=$ $\left[\begin{array}{lll}a & 0 & 0 \\ 0 & 0 & 0 \\ 0 & 0 & 0\end{array}\right], B=\left[\begin{array}{lll}b & 0 & 0 \\ 0 & 0 & 0 \\ 0 & 0 & 0\end{array}\right] \in L_{(0, t)}(R)$. By Lemma $5, B \in L_{(0, t)}(R)^{q n i l}$. By supposition there exists $B^{\prime}=\left[\begin{array}{ccc}x & 0 & 0 \\ 0 & z & t u \\ 0 & 0 & v\end{array}\right] \in L_{(0, t)}(R)^{q n i l}$ such that $B A=A B^{\prime}$. It implies $b a=a x$. By Lemma $6(1)$, $x \in R^{q n i l}$. Hence $b a=a x \in a R^{q n i l}$. Thus $R^{q n i l} a \subseteq a R^{q n i l}$.

There are right qnil-duo rings $R$ such that the rings $L_{(s, t)}(R)$ need not be right qnil-duo as shown below.

Example 9. The ring $L_{(1,1)}\left(\mathbb{Z}_{4}\right)$ is not right qnil-duo.

Proof. Let $A=\left[\begin{array}{lll}0 & 0 & 0 \\ 1 & 2 & 1 \\ 0 & 0 & 3\end{array}\right] \in L_{(1,1)}\left(\mathbb{Z}_{4}\right)$ and $B=\left[\begin{array}{lll}2 & 0 & 0 \\ 1 & 2 & 3 \\ 0 & 0 & 2\end{array}\right] \in L_{(1,1)}\left(\mathbb{Z}_{4}\right)^{\text {gnil }}$. Assume that there exists $C=\left[\begin{array}{lll}x & 0 & 0 \\ y & z & v \\ 0 & 0 & u\end{array}\right] \in L_{(1,1)}\left(\mathbb{Z}_{4}\right)^{\text {qnil }}$ such that $B A=A C$. Then $B A=\left[\begin{array}{lll}0 & 0 & 0 \\ 2 & 0 & 3 \\ 0 & 0 & 2\end{array}\right]$ and $A C=\left[\begin{array}{ccc}0 & 0 & 0 \\ x+2 y & 2 z & 2 v+u \\ 0 & 0 & 3 u\end{array}\right] . B A=A C$ implies $3=2 v+u$ and $2=3 u$. These equations lead us to contradiction. Hence $L_{(1,1)}\left(\mathbb{Z}_{4}\right)$ is not right qnil-duo.

The rings $H_{(s, t)}(R)$. Let $R$ be a ring and $s, t \in C(R)$ be invertible in $R$. Let

$$
H_{(s, t)}(R)=\left\{\left[\begin{array}{lll}
a & 0 & 0 \\
c & d & e \\
0 & 0 & f
\end{array}\right] \in M_{3}(R) \mid a, c, d, e, f \in R, a-d=s c, d-f=t e\right\} .
$$

Then $H_{(s, t)}(R)$ is a subring of $M_{3}(R)$.

Lemma 7. Let $A=\left[\begin{array}{lll}a & 0 & 0 \\ c & d & e \\ 0 & 0 & f\end{array}\right], B=\left[\begin{array}{lll}x & 0 & 0 \\ y & z & u \\ 0 & 0 & v\end{array}\right] \in H_{(s, t)}(R)$. Then

(1) $A B=B A$ if and only if $a x=x a, d z=z d, f v=v f$.

(2) $A$ is invertible with inverse $B$ if and only if $a x=x a=1, d z=z d=1, f v=v f=1$.

(3) $A \in H_{(s, t)}(R)^{\text {qnil }}$ if and only if $a, d, f \in R^{q n i l}$.

Proof. (1) The necessity is clear. For the sufficiency, suppose that $a x=x a, d z=z d, f v=v f$. The matrix $A B$ has $c x+d y$ as $(2,1)$ entry, $d u+e v$ as $(2,3)$ entry and $B A$ has $y a+z c$ as $(2,1)$ entry, $z e+u f$ as $(2,3)$ entry. To show $A B=B A$ it is enough to get $c x+d y=y a+z c$ and $d u+e v=z e+u f$. Now $s c x+s d y=a x+d(s y-x)=a x-d z=x a-z a+z a-d z=s y a+s z c$. So, $c x+d y=y a+z c$ since $s$ is invertible. Similarly, we get $d u+e v=z e+u f$.

(2) One way is clear. Assume that $a x=x a=1, d z=z d=1, f v=v f=1$. Let $B \in H_{(s, t)}(R)$ with $y=-z c x$ and $u=-z e v$. Then $A B=B A=I_{3}$. 
(3) Assume $A \in H_{(s, t)}(R)^{\text {gnil }}$. Let $x \in \operatorname{comm}(a), y \in \operatorname{comm}(f)$ and $D=\left[\begin{array}{ccc}x & 0 & 0 \\ s^{-1} x & 0 & -t^{-1} y \\ 0 & 0 & y\end{array}\right]$. Then $D \in \operatorname{comm}(A)$. In fact, $s c x=(a-d) x$ and tey $=(d-f) y$. Hence $I_{3}+A D$ is invertible in $H_{(s, t)}(R)$. It follows that $1+a x, 1+f y \in U(R)$. So, $a, f \in R^{q n i l}$. As for $d \in R^{q n i l}$, let $r \in \operatorname{comm}(d)$ and $D=\left[\begin{array}{ccc}0 & 0 & 0 \\ -s^{-1} r & r & t^{-1} r \\ 0 & 0 & 0\end{array}\right]$. Then $D \in \operatorname{comm}(A)$. By assumption $I_{3}+A D \in$ $U\left(H_{(s, t)}(R)\right)$. Hence $1+d r \in U(R)$. Hence $d \in R^{q n i l}$. Conversely, suppose that $a, d, f \in R^{q n i l}$. Let $B \in \operatorname{comm}(A)$. Then $x \in \operatorname{comm}(a), z \in \operatorname{comm}(d)$ and $v \in \operatorname{comm}(f)$. By supposition, $1+a x, 1+d y$ and $1+f v$ are invertible. By part (2), $I_{3}+A B \in U\left(H_{(s, t)}(R)\right)$. Hence $A \in$ $H_{(s, t)}(R)^{\text {gnil }}$. This completes the proof.

Theorem 11. Let $R$ be a ring. Then $R$ is right qnil-duo if and only if $H_{(s, t)}(R)$ is right qnil-duo.

Proof. Assume that $R$ is a right qnil-duo ring. Let $A=\left[\begin{array}{lll}a & 0 & 0 \\ c & d & e \\ 0 & 0 & f\end{array}\right] \in H_{(s, t)}(R)$ and $B=\left[\begin{array}{lll}x & 0 & 0 \\ y & z & u \\ 0 & 0 & v\end{array}\right] \in H_{(s, t)}(R)^{q n i l}$. By Lemma 7, $x, z, v \in R^{q n i l}$. There exist $x^{\prime}, z^{\prime}, v^{\prime} \in R^{q n i l}$ such that $x a=a x^{\prime}, z d=d z^{\prime}, v f=f v^{\prime}$. Let $y^{\prime}=s^{-1}\left(x^{\prime}-z^{\prime}\right)$ and $u^{\prime}=t^{-1}\left(z^{\prime}-v^{\prime}\right)$ and $B^{\prime}=\left[\begin{array}{ccc}x^{\prime} & 0 & 0 \\ y^{\prime} & z^{\prime} & u^{\prime} \\ 0 & 0 & v^{\prime}\end{array}\right]$. Then $B^{\prime} \in H_{(s, t)}(R)^{\text {qnil }}$. We next show that $B A=A B^{\prime}$. It is enough to see $y a+z c=c x^{\prime}+d y^{\prime}$ and $z e+u f=d u^{\prime}+e v^{\prime}$. We start with, $c x^{\prime}+d y^{\prime}=c x^{\prime}+d s^{-1} x^{\prime}-d s^{-1} z^{\prime}$. Multiplying the latter from the left by $s$ and using the fact that $s$ is central, we have

$$
\begin{aligned}
s\left(c x^{\prime}+d y^{\prime}\right)=s c x^{\prime}+d x^{\prime}-d z^{\prime} & =(s c+d) x^{\prime}-z d=a x^{\prime}-z d=x a-z d \\
& =(x a-z a)+(z a-z d)=s y a+s z c=s(y a+z c) .
\end{aligned}
$$

Since $s$ is invertible, $y a+z c=c x^{\prime}+d y^{\prime}$. Similarly, $d u^{\prime}+e v^{\prime}=d t^{-1} z^{\prime}-d t^{-1} v^{\prime}+e v^{\prime}$. Multiplying the latter from the left by $t$ and using the fact that $t$ is central, we have

$$
\begin{aligned}
t\left(d u^{\prime}+e v^{\prime}\right)=d z^{\prime}-d v^{\prime}+t e v^{\prime} & =z d+(t e-d) v^{\prime}=z d-f v^{\prime}=z d-v f \\
& =z d-z f+z f-v f=z(d-f)+(z-v) f=t(z e+u f) .
\end{aligned}
$$

By using invertibility of $t$, we get $d u^{\prime}+e v^{\prime}=z e+u f$. Conversely, suppose that $H_{(s, t)}(R)$ is a right qnil-duo ring. Let $a \in R$ and $b \in R^{\text {qnil }}$. Consider $A=a I_{3}, B=b I_{3} \in H_{(s, t)}(R)$. By Lemma 7, $B \in H_{(s, t)}(R)^{q n i l}$. By supposition, there exists $B^{\prime}=\left[\begin{array}{lll}x & 0 & 0 \\ y & z & u \\ 0 & 0 & v\end{array}\right] \in H_{(s, t)}(R)^{\text {gnil }}$ such that $B A=A B^{\prime}$. It implies $b a=a x$. Again by Lemma $7, x \in R^{q n i l}$. Hence $b a=a x \in a R^{q n i l}$. Thus $R^{\text {qnil }} a \subseteq a R^{q n i l}$. 
Generalized matrix rings. Let $R$ be a ring and $s \in U(R)$. Then $\left[\begin{array}{ll}R & R \\ R & R\end{array}\right]$ becomes a ring denoted by $K_{s}(R)$ with addition defined componentwise and multiplication defined in [10] by

$$
\left[\begin{array}{ll}
a_{1} & x_{1} \\
y_{1} & b_{1}
\end{array}\right]\left[\begin{array}{ll}
a_{2} & x_{2} \\
y_{2} & b_{2}
\end{array}\right]=\left[\begin{array}{cc}
a_{1} a_{2}+s x_{1} y_{2} & a_{1} x_{2}+x_{1} b_{2} \\
y_{1} a_{2}+b_{1} y_{2} & s y_{1} x_{2}+b_{1} b_{2}
\end{array}\right]
$$

In [10], $K_{s}(R)$ is called a generalized matrix ring over $R$.

Lemma 8. Let $R$ be a ring. Then the following hold.

(1) $U\left(K_{0}(R)\right)=\left\{\left[\begin{array}{ll}a & b \\ c & d\end{array}\right] \in K_{0}(R) \mid a, d \in U(R)\right\}$.

(2) $C\left(K_{0}(R)\right)=\left\{\left[\begin{array}{ll}a & 0 \\ 0 & a\end{array}\right] \in K_{0}(R) \mid a \in C(R)\right\}$.

Proof. (1) Let $A=\left[\begin{array}{ll}a & b \\ c & d\end{array}\right] \in U\left(K_{0}(R)\right)$. There exists $B=\left[\begin{array}{ll}x & y \\ z & t\end{array}\right] \in K_{0}(R)$ such that $A B=$ $B A=I$, where $I$ is the identity matrix. Then we have $a x=x a=1$ and $d t=t d=1$. So, $a$ and $d$ are invertible. Conversely, let $A=\left[\begin{array}{ll}a & b \\ c & d\end{array}\right] \in K_{0}(R)$ with $a, d \in U(R)$. Let $x=a^{-1}, t=d^{-1}$, $k=-a^{-1} b d^{-1}$ and $l=-d^{-1} c a^{-1}$. Then $B=\left[\begin{array}{ll}x & k \\ l & t\end{array}\right]$ is the inverse of $A$ in $K_{0}(F)$.

(2) Let $A=\left[\begin{array}{ll}a & b \\ c & d\end{array}\right] \in C\left(K_{0}(R)\right)$. By commuting $A$ in turn with the matrices $\left[\begin{array}{ll}1 & 0 \\ 0 & 0\end{array}\right]$ and $\left[\begin{array}{ll}0 & 1 \\ 0 & 0\end{array}\right]$ in $K_{0}(R)$ we reach at $A=\left[\begin{array}{ll}a & 0 \\ 0 & a\end{array}\right]$. For the converse, let $A=\left[\begin{array}{ll}a & 0 \\ 0 & a\end{array}\right] \in K_{0}(R)$, where $a \in C(R)$. Then clearly, $A$ commutes with every element of $K_{0}(R)$. So, $A \in C\left(K_{0}(R)\right)$.

Proposition 7. Let $R$ be a ring and $A=\left[\begin{array}{ll}a & b \\ c & d\end{array}\right] \in K_{0}(R)$. If $a, d \in R^{\text {qnil }}$, then $A \in K_{0}(R)^{\text {qnil }}$.

Proof. Suppose that $a, d \in R^{\text {qnil }}$. Let $B=\left[\begin{array}{ll}x & y \\ z & t\end{array}\right] \in K_{0}(R)$ with $B \in \operatorname{comm}(A)$. Then $x \in \operatorname{comm}(a), t \in \operatorname{comm}(d)$. Let $r=1+a x, v=1+d t, s=a y+b t$ and $u=c x+d z$. By assumption, $r=1+a x$ and $v=1+d t$ are invertible in $R$. Let $k=-r^{-1} s v^{-1}$ and $l=-v^{-1} u r^{-1}$. Then $I_{2}+A B=\left[\begin{array}{ll}r & s \\ u & v\end{array}\right]$ is invertible with the inverse $C=\left[\begin{array}{cc}r^{-1} & k \\ l & v^{-1}\end{array}\right]$.

Let $R$ be a ring, $a, b \in R$. Define $l_{a}-r_{b}: R \rightarrow R$ by $\left(l_{a}-r_{b}\right)(r)=a r-r b$ and $l_{b}-r_{a}: R \rightarrow R$ by $\left(l_{b}-r_{a}\right)(r)=b r-r a$. In [1], a local ring $R$ is called bleached if for any $a \in J(R)$ and any $b \in U(R)$, the abelian group endomorphisms $l_{b}-r_{a}$ and $l_{a}-r_{b}$ of $R$ are surjective. Such rings are called uniquely bleached if the appropriate maps are injective as well as surjective. In [13], $R$ is a weakly bleached ring provided that for any $a \in J(R), b \in 1+J(R), l_{a}-l_{b}$ and $l_{b}-l_{a}$ are surjective and it is proved that matrices over 2-projective free rings are strongly J-clean. It is proved that all upper triangular matrices over bleached local rings are strongly clean. In [12, Example 2] and [1, Theorem 18] it is proved that a local ring $R$ is weakly bleached if and only if the $2 \times 2$ upper triangular matrix ring $U_{2}(R)$ is strongly clean. In the preceding, the maps of the form $l_{a}-r_{b}$ play a central role. In this vein, we make use of the abelian group endomorphisms $l_{a}-r_{b}$ to get the following result as partly the converse of Proposition 7. 
Theorem 12. Let $R$ be a ring and $A=\left[\begin{array}{ll}a & b \\ c & d\end{array}\right] \in K_{0}(R)^{\text {qnil }}$. If for any $x \in \operatorname{comm}(a)$ and $y \in \operatorname{comm}(d)$ and for the abelian group endomorphisms $l_{y}-r_{x}$ and $l_{x}-r_{y}, b \in \operatorname{Ker}\left(l_{x}-r_{y}\right)$ and $c \in \operatorname{Ker}\left(l_{y}-r_{x}\right)$, then $a, d \in R^{q n i l}$.

Proof. Assume that $A=\left[\begin{array}{ll}a & b \\ c & d\end{array}\right] \in K_{0}(R)^{\text {qnil }}, x \in \operatorname{comm}(a)$ and $y \in \operatorname{comm}(d)$, for $l_{y}-r_{x}$ and $l_{x}-r_{y}, b \in \operatorname{Ker}\left(l_{x}-r_{y}\right)$ and $c \in \operatorname{Ker}\left(l_{y}-r_{x}\right)$. Then $b \in \operatorname{Ker}\left(l_{x}-r_{y}\right)$ implies $\left(l_{x}-r_{y}\right)(b)=0$. So, $x b=b y$. $c \in \operatorname{Ker}\left(l_{y}-r_{x}\right)$ implies $\left(l_{y}-r_{x}\right)(c)=0$. So, $y c=c x$. Let $B=\left[\begin{array}{ll}x & 0 \\ 0 & y\end{array}\right] \in K_{0}(R)$. Then $x b=b y$ and $y c=x c$ give rise to $B \in \operatorname{comm}(A)$. By hypothesis, $I_{2}+A B$ is invertible. Then Lemma 8 implies $1-a x$ and $1-d y$ are invertible. Hence $a, d \in R^{q n i l}$.

We may determine the set $K_{0}(R)^{\text {qnil }}$ for some rings $R$.

Proposition 8. (1) If $R$ is a local ring, then $A=\left[\begin{array}{ll}a & b \\ c & d\end{array}\right] \in K_{0}(R)^{\text {gnil }}$ if and only if $a, d \in R^{\text {qnil }}$.

(2) Let $R$ be a ring. Then $A=\left[\begin{array}{ll}a & 0 \\ 0 & d\end{array}\right] \in K_{0}(R)^{\text {gnil }}$ if and only if $a, d \in R^{\text {qnil }}$.

Proof. (1) Assume that $R$ is a local ring and $A=\left[\begin{array}{ll}a & b \\ c & d\end{array}\right] \in K_{0}(R)^{\text {qnil }}$, and $d \notin R^{q n i l}$. By Proposition $1, d \in U(R)$. In this case, $1+d$ can not belong to $U(R)$. By Lemma $8, I+A$ can not belong to $U\left(K_{0}(R)\right)$. This contradicts $A \in K_{0}(R)^{\text {gnil }}$. It follows that $d \in R^{\text {qnil }}$. Similarly, we obtain $a \in R^{\text {qnil }}$. The converse is clear by Proposition 7. Proof of (2) is clear.

There are some classes of rings $R$ in which $K_{0}(R)$ being a right qnil-duo ring implies $R$ being a right qnil-duo ring.

Theorem 13. Let $R$ be a ring. Then $K_{0}(R)$ being a right qnil-duo ring implies $R$ being a right qnil-duo ring if $R$ is one of the following rings.

(1) $R$ is local.

(2) $R$ has no nonzero zero divisors.

Proof. (1) Let $R$ be a local ring. Assume that $K_{0}(R)$ is a right qnil-duo ring. Let $a \in R, b \in R^{q n i l}$. Consider $A=\left[\begin{array}{ll}a & 0 \\ 0 & a\end{array}\right], X=\left[\begin{array}{ll}b & 0 \\ 0 & b\end{array}\right] \in K_{0}(R)$. By Proposition $7, X \in K_{0}(R)^{\text {qnil }}$. There exists $X^{\prime}=\left[\begin{array}{cc}x^{\prime} & y^{\prime} \\ z^{\prime} & t^{\prime}\end{array}\right] \in K_{0}(R)^{\text {qnil }}$ such that $X A=A X^{\prime}$. Hence $b a=a x^{\prime}$. By Proposition $8, x^{\prime} \in R^{q n i l}$. So, $b a=a x^{\prime} \in a R^{q n i l}$.

(2) Let $R$ be a ring having no nonzero zero divisors. Assume that $K_{0}(R)$ is a right qnil-duo ring. Let $a \in R, b \in R^{\text {qnil }}$. If $a=0$ or $b=0$, there is nothing to do. Let $a \neq 0$ and $b \neq 0$ and consider $A=\left[\begin{array}{ll}a & 0 \\ 0 & a\end{array}\right], B=\left[\begin{array}{ll}b & 0 \\ 0 & b\end{array}\right] \in K_{0}(R)$. By Proposition $7, B \in K_{0}(R)^{\text {qnil }}$. There exists $B^{\prime}=\left[\begin{array}{cc}x^{\prime} & y^{\prime} \\ z^{\prime} & t^{\prime}\end{array}\right] \in K_{0}(R)^{q n i l}$ such that $B A=A B^{\prime}$. It implies $b a=a x^{\prime}=a t^{\prime}, a y^{\prime}=0$ and $a z^{\prime}=0$. Hence $x^{\prime}=t^{\prime}$ and $y^{\prime}=z^{\prime}=0$. Hence $x^{\prime} \in R^{\text {qnil }}$ by Proposition 8 . 


\section{References}

[1] Borooah G., Diesl A.J., Dorsey T.J. Strongly clean triangular matrix rings over local rings. J. Algebra 2007, 312 (2), 773-797. doi:10.1016/j.jalgebra.2006.10.029

[2] Chung Y.W., Lee Y. Structures concerning group of units. J. Korean Math. Soc. 2017, 54 (1), 177-191. doi:10.4134/JKMS.j150666

[3] Cui J. Quasinilpotents in rings and their applications. Turkish J. Math. 2018, 42 (5), 2847-2855. doi:10.3906/mat1706-79

[4] Feller E.H. Properties of primary noncommutative rings. Trans. Amer. Math. Soc. 1958, 89 (1), 79-91. doi: 10.1090/S0002-9947-1958-0098763-0

[5] Han J., Lee Y., Park S. Duo ring property restricted to group of units. J. Korean Math. Soc. 2015, 52 (3), 489-501. doi:10.4134/JKMS.2015.52.3.489

[6] Harte R.E. On quasinilpotents in rings. Panamer. Math. J. 1991, 1, 10-16.

[7] Kim D.H. Nilpotent-duo property on powers. Commun. Korean Math. Soc. 2018, 33 (4), 1103-1112. doi: 10.4134/CKMS.c170453

[8] Kim H.K., Kim N.K., Lee Y. Weakly duo rings with nil Jacobson radical. J. Korean Math. Soc. 2005, 42 (3), 457-470. doi:10.4134/JKMS.2005.42.3.457

[9] Kim D.H., Yun S.J. Normality on Jacobson and nil radicals. Commun. Korean Math. Soc. 2019, 34 (1), 127-136. doi:10.4134/CKMS.c180082

[10] Krylov P.A. Isomorphism of generalized matrix rings. Algebra Logic 2008, 47 (4), 258-262. doi:10.1007/s10469008-9016-y

[11] Lian H., Zeng Q. An extension of Cline's formula for a generalized Drazin inverse. Turkish J. Math. 2016, 40, 161-165. doi:10.3906/mat-1505-4

[12] Nicholson W.K. Lifting idempotents and exchange rings. Trans. Amer. Math. Soc. 1977, 229, 269-278. doi: 10.1090/S0002-9947-1977-0439876-2

[13] Sheibani M., Chen H., Bahmani R. Strongly J-clean matrices over 2-projective-free rings. arXiv:1409.3974v2 [math.RA].

[14] Vaserstein L.N. Bass's first stable range condition. J. Pure Appl. Algebra 1984, 34 (2-3), 319-330. doi: 10.1016/0022-4049(84)90044-6

[15] Xu L., Xue W. Structure of minimal non-commutative zero-insertive rings. Math. J. Okayama Univ. 1998, 40, 69-76.

[16] Ying Z., Chen J. On quasipolar rings. Algebra Colloq. 2012, 19 (4), 683-692. doi:10.1142/S1005386712000557

[17] Yu H.P. Stable range one for exchange rings. J. Pure Appl. Algebra 1995, 98, 105-109.

Received 07.09.2020

Revised 16.03.2021

Гарманчі А., Куртулмаз Й., Унгор Б. Ауальна властивість для кілець з перспективи квазінільпотентності // Карпатські матем. публ. - 2021. — Т.13, №2. - C. 485-500.

У цій статті ми зосереджуємось на дуальній властивості для кілець через квазінільпотентні елементи, що дає новий вид узагальнень комутативності. Ми називаємо цей вид кілець qnil-duo. Насамперед доведено деякі властивості квазінільпотентів у кільці. Потім множину квазінільпотентів застосовано до дуальної властивості кілець, з цієї точки зору ми вводимо і вивчаємо праві (відповідно ліві) qnil-duo кільця. Ми показуємо, шо це поняття не є ліво-право симетричним. Серед іншого доведено, шо якшо кільце $H(R ; \alpha)$ рядів Гурвіца $є$ правим qnil-duo, то $R$ $\epsilon$ правим qnil-duo. Кожне праве qnil-duo кільце $є$ абелевим. Праве qnil-duo кільце обміну має стабільний ранг 1.

Ключові слова і фрази: квазінільпотентний елемент, duo кільце, qnil-duo кільце. 